\title{
Study on differentially expressed genes related to defoliation traits in two alfalfa varieties based on RNA-Seq
}

\author{
Qiming Cheng ${ }^{1}$, Shiqie Bai ${ }^{2}$, Gentu Ge${ }^{1}$, Ping Li ${ }^{2}$, Liying Liu ${ }^{3}$, Chengdong Zhang ${ }^{4^{*}}$ and Yushan Jia ${ }^{1 *}$ (D)
}

\begin{abstract}
Background: Alfalfa (Medicago sativa) is a widely cultivated, essential commercial forage crop. The protein content in its leaves is the critical factor in determining the quality of alfalfa. Thus far, the understanding of the molecular mechanism of alfalfa defoliation traits remains unclear. The transcriptome database created by RNA-Seq is used to identify critical genes related to defoliation traits.

Results: In this study, we sequenced the transcriptomes of the Zhungeer variety (with easy leaf abscission) and WL319HQ variety (without easy leaf abscission). Among the identified 66,734 unigenes, 706 differentially expressed genes (DEGs) upregulated, and 392 unigenes downregulated in the Zhungeer vs WL319HQ leaf. KEGG pathway annotations showed that 8,414 unigenes were annotated to 87 pathways and contained 281 DEGs. Six DEGs belonging to the "Carotenoid biosynthesis", "Plant hormone signal transduction" and "Circadian rhythm-plant" pathways involved in defoliation traits were identified and validated by RT-qPCR analyses.

Conclusions: This study used RNA-Seq to discover genes associated with defoliation traits between two alfalfa varieties. Our transcriptome data dramatically enriches alfalfa functional genomic studies. In addition, these data provide theoretical guidance for field production practice and genetic breeding, as well as references for future study of defoliation traits in alfalfa.
\end{abstract}

Keywords: Alfalfa, Transcriptome, Differentially expressed genes (DEGs), Defoliation

\section{Background}

Alfalfa (Medicago sativa L.) is a member of the plant family Fabaceae. Alfalfa is widely distributed across the world and cultivated as a commercial forage crop due to its high protein, vitamin and mineral content $[1,2]$. Mature alfalfa leaves are the primary crude protein storage organs (approximately $260 \mathrm{~g} \mathrm{~kg}^{-1}$ dry basis), and protein content is a crucial indicator of the quality of alfalfa hay [3]. However, the leaves fall off very easily due to external pressure during harvest [4], and the resulting low-quality hay cannot satisfy livestock nutritional requirements.

\footnotetext{
* Correspondence: z3201256@zmail.unsw.edu.au; jys_nm@sina.com ${ }^{4}$ School of Biotechnology and Biomolecular Sciences, University of New South Wales, Randwick, NSW 2052, Australia

${ }^{1}$ College of Grassland Resources and Environment, Key Laboratory of Forage Cultivation, Processing and High Efficient Utilization of the Ministry of Agriculture and Key Laboratory of Grassland Resources of the Ministry of Education, Inner Mongolia Agricultural University, Hohhot 010011, China Full list of author information is available at the end of the article
}

The main factors in leaf senescence are endogenous abscisic acid (ABA) and ethylene (ETH) [5, 6]. ABA is a natural plant hormone identified in the 1960s, with roles in promoting plant organ shedding and seed germination $[7,8]$. ABA is present in higher plant organs and tissues and especially abundant in mature and aging tissues or dormant organs. When plants are under biotic or abiotic stress, such as pathogen infection, drought or salinity, leaf senescence is induced by increasing ABA content [9-12]. ETH is another early-discovered plant hormone with function in the regulation of plant growth, such as the promotion of fruit ripening, acceleration of organ aging and shedding [13]. ETH is synthesized by conversion from methionine with enough oxygen, which occurs in various plant tissues and organs [14]. ETH synthesis is regulated by a variety of growth signals with tissue specificity at different development stages, and the accumulation of ETH can also stimulate further ETH production [15]. The content of ETH is

(c) The Author(s). 2018 Open Access This article is distributed under the terms of the Creative Commons Attribution 4.0 International License (http://creativecommons.org/licenses/by/4.0/), which permits unrestricted use, distribution, and 
significantly higher in the processes of fruit ripening, seed germination, and leaf and flower aging off $[16,17]$. In addition, the synthesis of ETH is affected by a variety of external factors, such as mechanical damage, pathogen infection, low temperature, drought and salinity [18].

Previous studies showed that both ABA and ETH have the function of accelerating plant organ shedding. However, the physiological mechanisms are still unclear and remain controversial [19]. A study by Addicott [20] showed that endogenous $\mathrm{ABA}$ had positive effects on plant organ shedding, whereas field experiments failed, possibly because indoleacetic acid (IAA), gibberellin (GA) and cytokinin (CTK) in leaves have an adverse effect on ABA. Furthermore, the effect of introducing exogenous $\mathrm{ABA}$ on plant organ abscission was weaker than the effect of exogenous ETH. Osborne [21] concluded that ABA caused the premature senescence of organ cells, but that this may be due to increasing ETH content in plant organs that leads to organ shedding.

Transcriptome refers to a collection of all the transcription products under a specific physiological condition, including messenger RNA, ribosomal RNA, transport RNA and noncoding RNA [22]. Transcriptome sequencing research is essential for novel gene discovery, functional gene annotation, gene differential expression and the development of molecular markers [23-25]. Next Generation Sequencing (NGS) technology enables complete and rapid access to almost all transcript sequence information of a particular tissue or organ of a species and has been widely used in modal and nonmodel plant research [2628]. To our knowledge, there have been no previous reports of research on DEGs related to defoliation traits in alfalfa varieties. This study aimed to construct a transcriptome database of two alfalfa leaf varieties in the early blooming stage. Based on DEGs, we aimed to identify the critical genes related to defoliation traits in the Zhungeer variety (characterized by easy leaf abscission) and the WL319HQ variety (without easy leaf abscission). The results of this study provide a theoretical basis for future alfalfa molecular breeding and provide a reference for subsequent study of the transcription of alfalfa leaves.

\section{Results}

\section{De novo assembly transcriptome}

Six libraries of the total RNA extracted from the leaves of Zhungeer and WL319HQ varieties at the early flowering stage were constructed for high-throughput sequencing. A total of $261,775,004$ clean reads with a total of 39,202,954,927 nucleotides (nt) were obtained from the six sequencing libraries. Trinity method assembled a total of 66,734 unigenes with an N50 of 1496 nt. The maximum, minimum and average lengths were 143,789 nt, $201 \mathrm{nt}$ and 869 nt, respectively (Fig. 1).

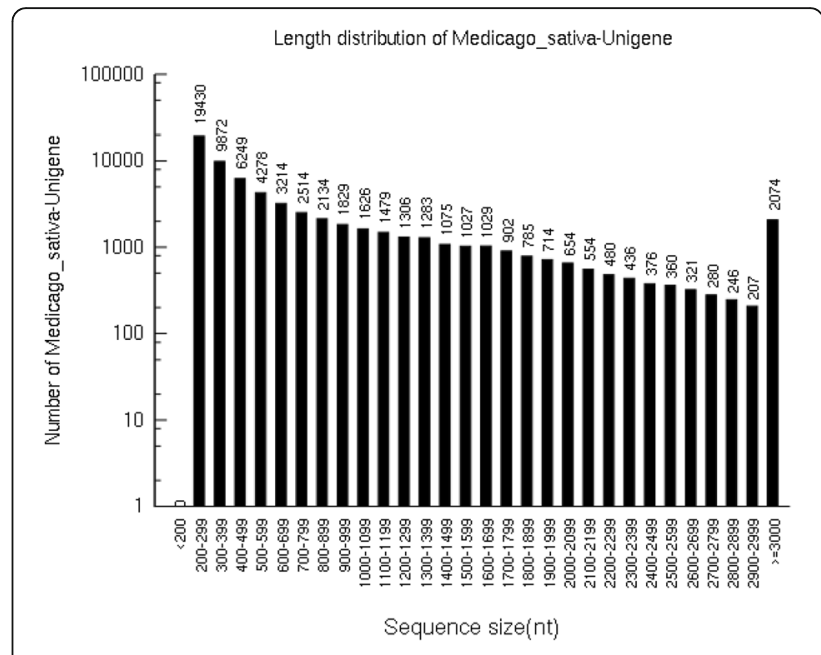

Fig. 1 The size distribution of M. sativa unigenes. The abscissa is the length of the assembled unigenes from $200 \mathrm{nt}$ to $\geq 3000 \mathrm{nt}$, and the ordinate is the number of unigenes of the corresponding length

\section{Gene annotation}

A total of 45,657 unigenes $(68.42 \%$ of total 66,734 unigenes) were annotated against Nr, Swiss-Prot, KOG and KEGG databases using BLASTx (E-value $<1 \times 10^{-5}$ ). Among them, 42,888 (67.26\%), 29,190 (43.74\%), 24,844 (37.23\%) and 15,647 (23.45\%) unigenes were annotated to the Nr, Swiss-Prot, KOG and KEGG databases, respectively (Fig. 2a). According to the $\mathrm{Nr}$ database, 19.26\% of unigenes showed homology $\left(1 \times \mathrm{E}^{-20}<\right.$ E-value $\left.\leq 1 \times \mathrm{x} \mathrm{E}^{-5}\right), 42.48 \%$ of unigenes showed strong homology $\left(1 \times \mathrm{E}^{-100}<\mathrm{E}\right.$-value $\leq 1 \mathrm{E}^{-20}$ ), and the remaining of $38.26 \%$ of unigenes had very strong homology (E-value $\leq 1 \mathrm{E}^{-100}$ ) (Fig. $2 \mathrm{~b}$ ). For the species distribution of the top BLAST hits, 3,441 unigenes matched to the homologous sequences of Medicago truncatula, while 2,679, 1,058 and 551 unigenes matched to the homologous sequences of Cicer arietinum, Cajanus cajan and Glycine max, respectively (Fig. 2c).

\section{Identification and analysis of DEGs}

Using the $\mathrm{Nr}$ annotation results, Blast2GO software was used to analyze GO functional annotations of unigenes, and WEGO software was used to perform the functional classification of unigenes. A total of 1,098 significant DEGs were assigned to one or more ontologies by the standard of $|\log 2 \mathrm{FC}|>1$ and FDR $<0.05$ (Additional file 1: Table S1; Additional file 2: Table S2; Fig. 3), 706 unigenes were upregulated, and the other 392 unigenes were downregulated (Zhungeer vs WL319HQ). The significant DEGs were used for the subsequent analysis.

In this group, "biological process", "metabolic process", "cellular process", "response to stimulus" and "single-organism process" were the most frequent terms and contained 288, 260, 174 and 159 unigenes, respectively. For "metabolic process", there were 195 upregulation unigenes 

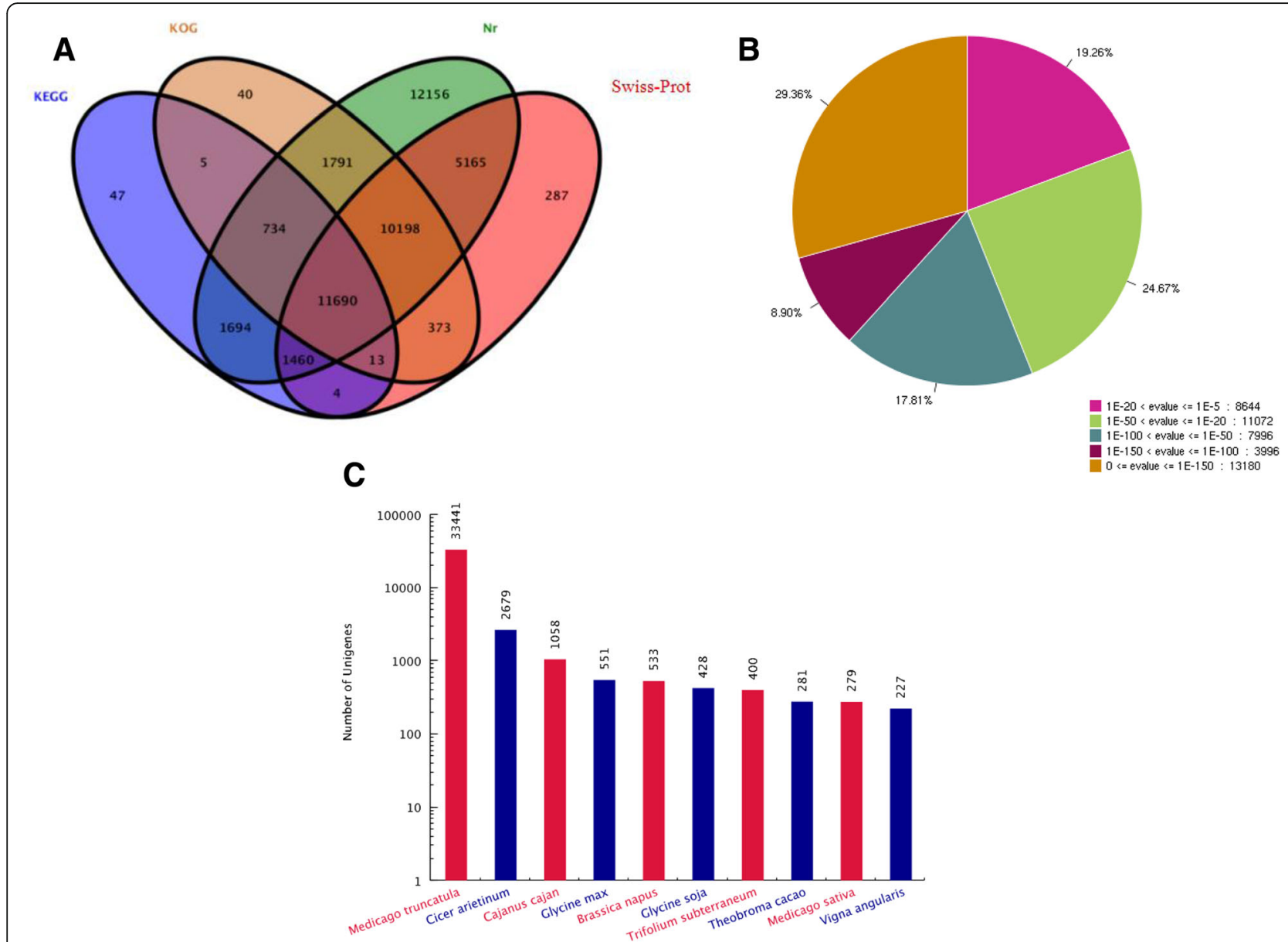

Fig. 2 Homology search of M. sativa unigenes. a Venn diagram of unigene numbers annotated by BLASTx with a cut-off E-value of $1 \times 10^{-5}$ against protein databases. The numbers in the circles indicate the number of unigenes annotated by single or multiple databases. $\mathbf{b}$ E-value distribution of the top BLASTx hits against the Nr database. $\mathbf{c}$ Ten top BLASTx hits of homologous sequences for the species distribution

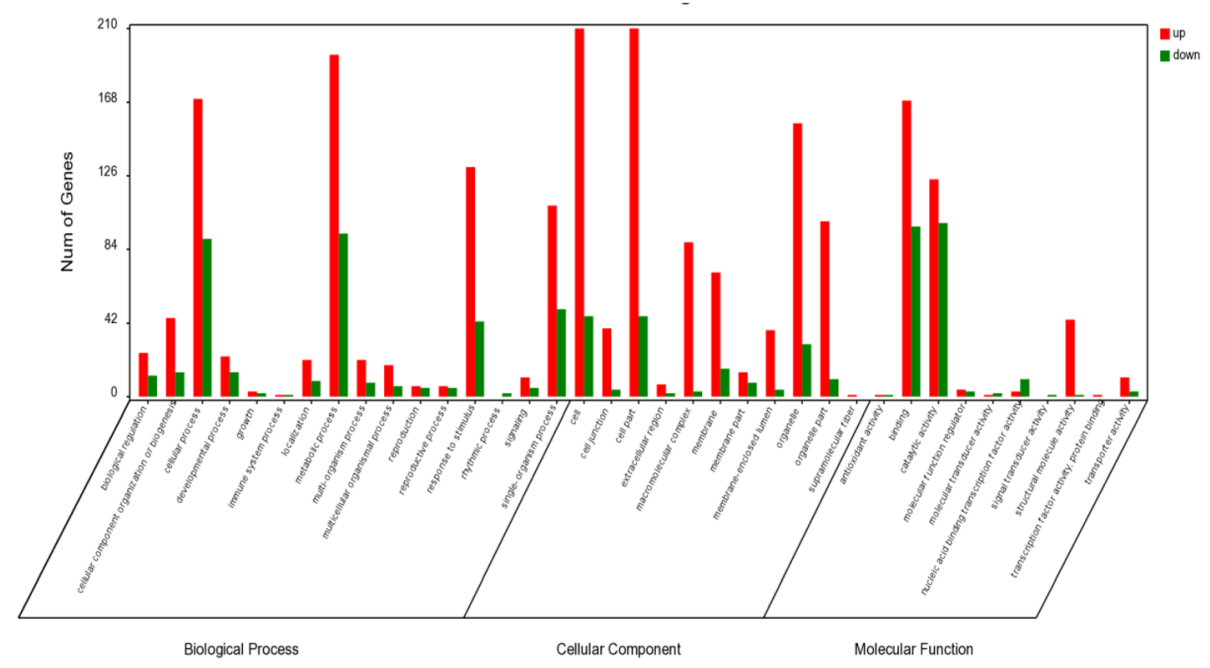

Fig. 3 GO classification of assembled unigenes. A total of 17,156 unigenes were categorized into three main categories: biological process, cellular component and molecular function 
and 93 downregulation unigenes. For "cellular process", there were 170 upregulation unigenes and 90 downregulation unigenes. For "response to stimulus", there were 131 upregulation unigenes and 43 downregulation unigenes. For "single-organism process", there were 109 upregulation unigenes and 50 downregulation unigenes, whereas "immune system process" (two unigenes) and "rhythmic process" (two unigenes) were infrequent. Under the "cellular component" group, most of those were classified into "cell" (210 upregulation unigenes, 46 downregulation unigenes), "cell part" (210 upregulation unigenes, 46 downregulation unigenes), and "organelle" (156 upregulation unigenes, 30 downregulation unigenes). For the "molecular function" group, "binding" (169 upregulation unigenes, 97 downregulation unigenes) and "catalytic activity" (124 upregulation unigenes, 99 downregulation unigenes) were the most abundant subcategories.

\section{KEGG pathway enrichment analysis}

A total of 8414 unigenes were assigned to 87 KEGG pathways (Additional file 3: Table S3). The 15 top KEGG pathways with the highest representation of DEGs were: "Ribosome" (ko03010), "Spliceosome" (ko03040), "Protein processing in endoplasmic reticulum" (ko0414), "Carbon metabolism" (ko01200), "Endocytosis" (ko04144), "Biosynthesis of amino acids" (ko01230), "RNA transport" (ko03013), "Plant-pathogen interaction" (ko04626), "Starch and sucrose metabolism" (ko00500), "Oxidative phosphorylation" (ko00190), "Nucleotide excision repair" (ko03420), "Plant hormone signal transduction" (ko04075), "DNA replication" (ko0303), "Ubiquitin-mediated proteolysis" (ko04120), and "Homologous recombination" (ko03440). $622(14.58 \%)$ unigenes and 64 DEGs accounting for $22.78 \%$ of 281 DEGs were annotated to the "Ribosome" pathway.

In the 87 KEGG pathways, the DEGs related to direct or indirect effects on the content of ABA and ETH were predicted. In the "Plant hormone signal transduction" pathway (ko04075), 294 unigenes with 6 DEGs were annotated. In the "Circadian rhythm-plant" pathway (ko04712), 61 unigenes with 4 DEGs were annotated. In addition, the "Carotenoid biosynthesis" pathway (ko00906) contained 44 unigenes with 1 DEG crucial for ABA synthesis, which was annotated.

\section{Real-time quantitative PCR analysis of DEGs related to defoliation}

To test the reliability of transcriptome sequencing data in the three pathways, Unigene0044746 (Auxin response factor, $A R F$ ), Unigene0002039 (Phytochrome interacting factor 3, PIF3), Unigene0027311 (Ethylene receptor protein, ETR), Unigene0053251 (Phytochrome B, PHYB), Unigene0053032 (Cryptochrome, CRY) and Unigene0014585 (9-cis-epoxycarotenoid dioxygenase 3, NCED3) (Additional file 4: Table
S4) were selected for One-Step RT-qPCR analysis. In addition to DEGs, Unigene0030464 (Tubulin alpha, TUBA) and Unigene0032887 (indole-3-acetic acid-amido synthetase, $G H$ ) were selected in the RT-qPCR analysis as unaffected genes. The alfalfa GAPDH gene was used as the endogenous reference. The significant difference of RTqPCR data between two varieties was analyzed by t-test, and the expression patterns revealed by RT-qPCR analysis were similar to those revealed by transcriptome sequencing for the same genes (Fig. 4).

\section{Discussion}

ABA, ETH, jasmonic acid, salicylic acid, CTK and auxin are the primary phytohormones that regulate plant senescence [29]. When plants are under environmental stress, such as drought, salinity or low temperature, the leaves will accelerate senescence and abscission due to the elevation of ABA concentration [11, 30]. In addition, ETH stimulates the synthesis of cellulase and controls the release of cellulase from protoplasts into the cell wall, which promotes cell wall degradation and leaf abscission [5]. Many studies reported that ETH levels would rise if plant tissues were damaged by mechanical force $[4,31,32]$. Therefore, the abscission of alfalfa leaves may be due to mechanical damage to plant tissues during harvesting, resulting in elevation of ETH concentration in leaf tissues. The mechanism by which phytohormone interactions promote the abscission of leaves is not well understood and is difficult to study [33].

In higher plants, xanthoxin is not only the synthesis precursor of ABA but also a metabolic intermediate of carotenoid catabolism [34]. The RNA-Seq data revealed that the alfalfa 9-cis-epoxycarotenoid dioxygenase 3 (NCED3) gene differentially expressed between WL319HQ and Zhungeer varieties. The RT-qPCR analysis showed that the transcription of the Zhungeer variety was approximately 2.3 times higher than the WL319HQ variety. $N C E D$ is the enzyme that catalyzes the cleavage reaction of 9-cis-epoxycarotenoids to produce xanthoxin and is the rate-limiting enzyme of ABA biosynthesis in higher plants [35-37]. NCED has been identified in maize, kidney bean, tomato and Arabidopsis thaliana [4, 35, 36, 38, 39]. A. thaliana NCED and its role in ABA biosynthesis are the most well-studied [37]. Overexpression of A. thaliana NCED3 resulted in a significantly higher level of endogenous ABA in the plant [40], and similar results were obtained in transgenic tobacco to overexpress the tomato NCED1 gene [38].

The "Plant hormone signal transduction" (ko04075) pathway plays a vital role in the metabolism of phytohormones in higher plants. In the RNA-Seq data, the expression level of the ethylene receptor protein (ETR) gene in Zhungeer variety was approximately 1.4 times higher than the WL319HQ variety. ETR is a protein that 


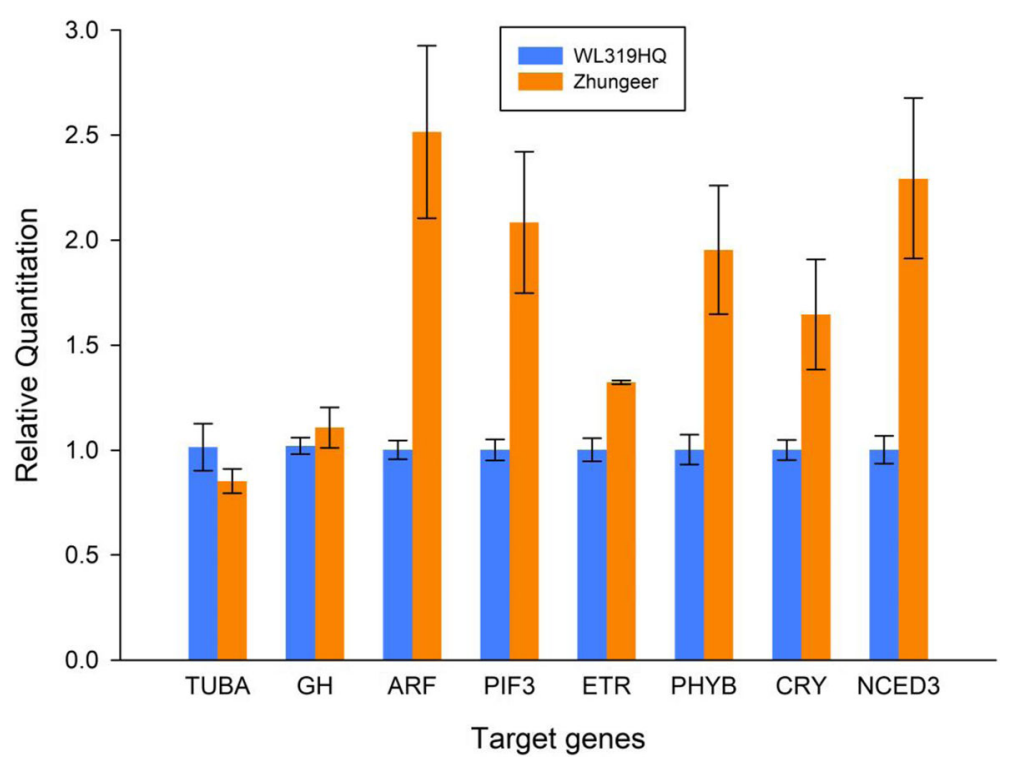

Fig. 4 The relative expression level of DEGs involved in "Plant hormone signal transduction", "Circadian rhythm-plant" and "Carotenoid biosynthesis" pathways revealed by RT-qPCR analysis. TUBA: Tubulin alpha, no significant difference ( $P=0.165)$; GH: indole-3-acetic acid-amido synthetase, no significant difference $(P=0.246)$; ARF: Auxin response factor, significant difference $(P=0.033)$; $P$ IF3: Phytochrome interacting factor 3 , significant difference $(P=0.043)$; ETR: Ethylene receptor protein, significant difference $(P=0.014)$; $P H Y B$ : Phytochrome $B$, significant difference $(P=0.047)$; CRY: Cryptochrome, significant difference $(P=0.049)$; NCED3: 9-cis-epoxycarotenoid dioxygenase 3, significant difference $(P=0.04)$

initiates the ETH signal transduction pathway by binding the ETH and relaying the ETH signal to constitutive triple response 1 (CTR1) Raf-like protein kinase [41]. The high-affinity binding activity has been confirmed in A. thaliana, as well as the tomato [42-44]. In the present study, the ETR gene was downregulated in WL319HQ compared to the Zhungeer variety, indicating that Zhungeer is more likely to accumulate ETH than the WL319HQ variety.

Auxin response factors $(A R F)$ are transcription factors that bind specifically to the DNA sequence 5'-TGTCTC-3' found in auxin-responsive promoter elements (AuxREs). Although ARF genes are not involved in leaf senescence directly, they act as transcriptional activators or repressors and therefore promote flowering, stamen development, floral organ abscission and fruit dehiscence [45]. Study of the $A$. thaliana ARF1 and ARF2 mutant has shown that $A R F 2$ may affect plant tissue shedding [46]. Nagpal et al. [47] found that the $A$. thaliana ARF6 gene promotes flower organ maturation and senescence. In addition, NPH4/ARF7 and ARF19 not only enhance the ARF2 phenotype but also induce leaf senescence [46]. The $A R F$ gene was significantly downregulated in alfalfa leaves in the WL319HQ variety.

Plant circadian rhythm is a hereditary physiological regulatory mechanism that synchronizes with circadian alternation in the plant. The response of plants to environmental factors mainly depends on the hormone regulatory network, including CTK, IAA, ABA and GA [48]. Circadian rhythm plays an essential role in the plant hormonal regulation pathway $[49,50]$. At the same time, the plant hormone signaling network also acts on circadian rhythm to transmit different metabolic signals and environmental signals to the endogenous circadian rhythm system, thus forming a complex regulatory network $[51,52]$. In this study, three DEGs involved in the plant circadian rhythm pathway were identified that may affect the regulation of ABA and ETH. Compared with Zhungeer, the PHYB (-1.775-fold), PIF3 (-3.715-fold) and $C R Y(-1.952)$ gene of WL319HQ were downregulated. PIF3 plays a critical role not only in light and temperature-mediated environmental signaling but also in the signaling of ABA and ETH. Light activates the phytochrome pathway to induce the degradation of PIF3 and promotes the accumulation of carotenoids, which may lead to an increase in ABA content.

In summary, it is likely that the Zhungeer alfalfa variety is more susceptible to abscission than the WL319HQ variety due to higher levels of plant hormone accumulation. Higher expression of NCED3 and PIF3 genes caused increasing $\mathrm{ABA}$ content, which may be a reason why the Zhungeer variety is more likely to defoliate. In addition, upregulation of ETR and ARF expression affects ETH; auxin accumulation is another indirect evidence as well.

\section{Conclusion}

This study is the first to use RNA-Seq to identify the defoliation traits between alfalfa varieties. It is essential to understand the physiological effects of various hormones 
on plants, the interaction between hormones and their relationship with the agricultural production environment. Our transcriptome data dramatically enriches alfalfa genome research. This research will provide theoretical guidance for field production practice and genetic breeding and provides a reference for the future study of defoliation traits in alfalfa.

\section{Methods}

\section{Sample collection}

We selected two varieties of the tetraploid M. sativa: the Zhungeer variety that has easy leaf abscission and the WL319HQ variety that is not characterized by easy leaf abscission. Both varieties were grown in experimental fields located in Baotou City, Inner Mongolia, China $\left(110^{\circ}\right.$ $\left.37^{\prime} \mathrm{E}, 40^{\circ} 5^{\prime} \mathrm{N}\right)$. Leaves of the Zhungeer and WL319HQ varieties were collected from two-year-old plants at the early flowering stage in June. All samples were immediately frozen in liquid nitrogen and stored at $-80{ }^{\circ} \mathrm{C}$ for future RNA extraction.

\section{RNA extraction and RNA-Seq library construction}

Total RNA was extracted from three biological replicates of the leaves of each $M$. sativa variety using the RNeasy Plant Mini Kit (Qiagen, Germany), following the manufacturer's protocol. The integrity of RNA samples was measured by Agilent 2100 Bioanalyzer (Agilent Technologies, USA). The Poly (A) mRNA was enriched by NEBNext Oligo $(\mathrm{dT})_{25}$ beads (NEB, USA) from $50 \mu \mathrm{l}$ total RNA. Then, the enriched mRNA was constructed to a cDNA library by NEBNext Ultra RNA Library Prep Kit for Illumina (NEB, USA), following the manufacturer's protocol.

\section{Sequencing and raw reads filtering}

The transcriptome sequencing was performed using an Illumina HiSeq 4000 sequencing platform with pairedend $150 \mathrm{bp}$ (PE150) sequencing strategy at Gene Denovo Biotechnology Co. (Guangzhou, China). Raw reads obtained from the sequencing that may affect the following assembly and analysis were removed, including reads with adapters, reads containing more than $10 \%$ unknown nucleotides and low-quality reads containing more than $50 \%$ low-quality bases (Q-values $\leq 10)$.

\section{De novo transcriptome assembly and unigenes detection}

Since $M$. sativa genome information was not previously available, full-length transcriptome de novo assembly was carried out using the reference genome independent Trinity method [53]. Trinity is a software package consisting of three modules: Inchworm, Chrysalis and Butterfly. First, Inchworm assembled clean reads to a collection of linear contigs by the greedy k-mer method. Second, the related minimal overlapping contigs created by Inchworm were clustered into connected components by Chrysalis, and a de Bruijn graph was constructed for each cluster of related contigs. Finally, Butterfly analyzed the paths from the de Bruijn graphs that were taken by reads and pairs of reads, and the complete transcripts and unigenes sets were obtained from the outputs [54].

\section{Unigenes annotation}

The unigenes were annotated using the BLASTx program (http://www.ncbi.nlm.nih.gov/BLAST/) with an E-value threshold of $1 \times 10^{-5}$ to $\mathrm{Nr}$ (NCBI nonredundant protein) database (http://www.ncbi.nlm.nih.gov), the Swiss-Prot (A manually annotated and reviewed protein sequence database) protein database (http://www.expasy.ch/sprot), the KEGG (Kyoto Encyclopedia of Genes and Genomes) database (http://www.genome.jp/kegg) and the KOG (Clusters of Orthologous Groups of proteins) database (http:// www.genome.jp/kegg/ko.html). Protein functional annotations were obtained according to the best alignment results.

\section{Analysis of DEGs}

Gene expression analysis of unigenes was performed and normalized by RPKM (reads per kb per million reads). The edgeR package (http://www.r-project.org/) was used to identify DEGs across samples or groups. Genes with a fold change $\geq 2$ and a false discovery rate $(F D R)<0.05$ were considered significant DEGs. DEGs were further subjected to GO functional enrichment analysis and KEGG pathway analysis.

\section{Real-time quantitative PCR analysis}

One-Step Real-time quantitative PCR (RT-qPCR) was carried out to confirm the RNA-Seq data from the extracted $M$. sativa leaves' total RNA. The experiments were performed using One-Step TB Green PrimeScript RT-PCR Kit II (Takara, Japan) on an ABI 7500 system (Applied Biosystems, USA). Primers for One-Step RTqPCR were designed using Primer Premier 5.0 software (Premier, Canada). The alfalfa GAPDH gene was selected as the endogenous reference [55]. All the primers were synthesized by Invitrogen (Beijing, China) and are listed in Additional file 5: Table S5. A $20 \mu \mathrm{l}$ reaction mix was set up containing $50 \mathrm{ng}$ total RNA and $0.4 \mu \mathrm{M}$ of each primer. The One-Step RT-qPCR analysis was performed on the extracted total RNA in three biological replicates of the leaves of each M. sativa variety, and each RNA sample was carried out in technical triplicate. The program was set at $42{ }^{\circ} \mathrm{C}$ for $5 \mathrm{~min}$ then $95{ }^{\circ} \mathrm{C}$ for $10 \mathrm{~s}$, followed by 40 cycles of $95{ }^{\circ} \mathrm{C}$ for $5 \mathrm{~s}$ then $60{ }^{\circ} \mathrm{C}$ for $34 \mathrm{~s}$. The final melting curve analysis was set at $95{ }^{\circ} \mathrm{C}$ for $15 \mathrm{~s}, 60{ }^{\circ} \mathrm{C}$ for $1 \mathrm{~min}$ and $95^{\circ} \mathrm{C}$ for $15 \mathrm{~s}$. The relative expression changes of the endogenous reference and tested genes were analyzed by the $2^{-\Delta \Delta C T}$ method [56]. 


\section{Statistical analysis}

Statistical analyses were performed using the software SigmaPlot 12.5 (Systat Software, Inc. USA). The ShapiroWilk test was used to assess the data for normality. Then, the t-test was used to test the expression levels of target genes between the Zhungeer and WL319HQ varieties. Results were considered significant at $5 \%$.

\section{Additional files}

Additional file 1: Table S1. DEGs generated from two varieties of alfalfa leaves. (DOCX $144 \mathrm{~kb}$ )

Additional file 2: Table S2. GO functional annotations and the number of DEGs statistics. (DOCX $18 \mathrm{~kb}$ )

Additional file 3: Table S3. KEGG pathway annotation of DEGs between two varieties of alfalfa leaves. (DOCX $26 \mathrm{~kb}$ )

Additional file 4: Table S4. Genes for RT-qPCR analysis and the list of GO ID. (DOCX 14 kb)

Additional file 5: Table S5. Primers used for RT-qPCR analysis. (DOCX $12 \mathrm{~kb}$ )

\section{Abbreviations}

ABA: Abscisic acid; ARF: Auxin response factor; AuxREs: Auxin-responsive promoter elements; BLAST: Basic Local Alignment Search Tool; CRY: Cryptochrome; CTK: Cytokinin; CTR1: Constitutive triple response 1; DEGs: Differentially expressed genes; ETH: Ethylene; ETR: Ethylene receptor protein; GA: Gibberellin; $G H$ : Indole-3-acetic acid-amido synthetase; GO: Gene Ontology; IAA: Indoleacetic acid; KEGG: Kyoto Encyclopedia of Genes and Genomes; KOG: Clusters of Orthologous Groups of proteins; NCED3: 9-cis-epoxycarotenoid dioxygenase 3; Nr: NCBI nonredundant protein; nt: Nucleotides; PHYB: Phytochrome B; PIF3: Phytochrome interacting factor 3; RT-qPCR: Real-time quantitative PCR; Swiss-Prot: A manually annotated and reviewed protein sequence database; TUBA: Tubulin alpha

\section{Acknowledgments}

We thank Gene Denovo Biotechnology Co. (Guangzhou, China) for providing technical support.

\section{Funding}

This study was supported by the National Natural Science Foundation of China (31572461). The funding body had no role in the design of the study, collection, analysis, or interpretation of data or in the writing of the manuscript.

\section{Availability of data and materials}

Our transcriptome datasets are available at NCBI project PRJNA472434 with accession number SRP148624, and SRA with accession number SRR7193615, SRR7193614, SRR7193613, SRR7193612, SRR7193611, SRR7193610.

\section{Authors' contributions}

YSJ and SQB designed the experiments and revised the manuscript. QMC, LYL performed the experiments. QMC, CDZ wrote the manuscript. GTG and $\mathrm{PL}$ carried out the data analysis. All authors reviewed, considered and approved the manuscript.

\section{Ethics approval and consent to participate}

The two alfalfa cultivars in this study were collected from Institute of Grassland Research of CAAS, located in Hohhot, Inner Mongolia, North China (latitude $40^{\circ}$ $48^{\prime} \mathrm{N}$, longitude $111^{\circ} 42^{\prime} \mathrm{E}$, elevation $1070 \mathrm{~m}$ ). No specific permit was required for the field collection of alfalfa cultivars and the location is not privately-owned or protected in any way.

\section{Consent for publication}

Not applicable.

\section{Competing interests}

The authors declare that they have no competing interests.

\section{Publisher's Note}

Springer Nature remains neutral with regard to jurisdictional claims in published maps and institutional affiliations.

\section{Author details}

${ }^{1}$ College of Grassland Resources and Environment, Key Laboratory of Forage Cultivation, Processing and High Efficient Utilization of the Ministry of Agriculture and Key Laboratory of Grassland Resources of the Ministry of Education, Inner Mongolia Agricultural University, Hohhot 010011, China. ${ }^{2}$ Sichuan Academy of Grassland Sciences, Chengdu 611731, China. ${ }^{3}$ Inner Mongolia Academy of Forestry Science, Hohhot 010010, China. ${ }^{4}$ School of Biotechnology and Biomolecular Sciences, University of New South Wales, Randwick, NSW 2052, Australia.

Received: 23 May 2018 Accepted: 17 October 2018

Published online: 07 November 2018

\section{References}

1. Yacoubi R, Job C, Belghazi M, Chaibi W, Job D. Toward characterizing seed vigor in alfalfa through proteomic analysis of germination and priming. J Proteome Res. 2011;10(9):3891-903.

2. Jia C, Wu X, Chen M, Wang Y, Liu X, Gong P, Xu Q, Wang X, Gao H, Wang Z. Identification of genetic loci associated with crude protein and mineral concentrations in alfalfa (Medicago sativa) using association mapping. BMC Plant Biol. 2017:17:97.

3. Hojilla-Evangelista MP, Selling GW, Hatfield R, Digman M. Extraction, composition, and functional properties of dried alfalfa (Medicago sativa L.) leaf protein. J Sci Food Agr. 2017;97(3):882-8.

4. Lieberman M. Biosynthesis and action of ethylene. Annu Rev Plant Biol. 1979;30:533-91.

5. Reid PD, Strong HG, Lew F, Lewis LN. Cellulase and abscission in the red kidney bean (Phaseolus vulgaris). Plant Physiol. 1974;53:732-7.

6. Bleecker AB, Patterson SE. Last Exit: Senescence, abscission, and meristem arrest in Arabidopsis. Plant Cell. 1997;9:1169-79.

7. Walker-Simmons M. ABA levels and sensitivity in developing wheat embryos of sprouting resistant and susceptible cultivars. Plant Physiol. 1987:84(1):61-6.

8. Finkelstein RR, Gampala SSL, Rock CD. Abscisic acid signaling in seeds and seedlings. Plant Cell. 2002;14(1):15-45.

9. Adie BAT, Pérez-Pérez J, Pérez-Pérez MM, Godoy M, Sánchez-Serrano J, Schmelz EA, Solano R. ABA is an essential signal for plant resistance to pathogens affecting JA biosynthesis and the activation of defenses in Arabidopsis. Plant Cell. 2007;19(5):1665-81.

10. Figueiredo MVB, Burity HA, Martínez CR, Chanway CP. Alleviation of drought stress in the common bean (Phaseolus vulgaris L.) by co-inoculation with Paenibacillus polymyxa and rhizobium tropici. Appl Soil Ecol. 2008; 40(1):182-8.

11. Zhang J, Liang S, Duan J, Wang J, Chen S, Cheng Z, Zhang Q, Liang X, Li Y. De novo assembly and characterisation of the transcriptome during seed development, and generation of genic-SSR markers in Peanut (Arachis hypogaea L.). BMC Genomics. 2012;13:90.

12. Lovelli S, Sofo A, Perniola M, Scopa A. Abscisic acid and biomass partitioning in tomato under salinity. In: Ahmad P, Azooz M, Prasad M, editors. Ecophysiology and responses of plants under salt stress. New York: Spinger; 2013. p. 267-82.

13. Burg SP. Ethylene in plant growth. PNAS. 1973;70(2):591-7.

14. Davies PJ. Ethylene in plant biology. Cell. 1993;72(1):11-2.

15. Reig C, Martínez-Fuentes A, Mesejo C, Rodrigo MJ, Zacarías L, Agustí M. Loquat fruit lacks a ripening-associated autocatalytic rise in ethylene production. J Plant Growth Regul. 2016;35(1):232-44

16. McMurchie EJ, McGlasson WB, Eaks IL. Treatment of fruit with propylene gives information about the biogenesis of ethylene. Nature. 1972;237:235-6.

17. Machabé ES, Saini HS. Differences in the requirement for endogenous ethylene during germination of dormant and non-dormant seeds of Chenopodium album L. J Plant Physiol. 1991;138(1):97-101.

18. Fluhr R, Mattoo AK, Dilley DR. Ethylene-biosynthesis and perception. Crit Rev Plant Sci. 1996;15(5\&6):479-523. 
19. Kitsaki CK, Vemmos SN, Tzoutzoukou CG. Changes of respiration rate, ethylene evolution, and abscisic acid content in developing inflorescence and young fruit of olive (Olea europaea L. cv. Konservolia). J Plant Growth Regul. 1999;18:1-7.

20. Addicott FT. Abscisic acid. New York: Praeger; 1983.

21. Osborne DJ, Morgan PW. Abscission. Crit Rev Plant Sci. 1989;8(2):103-29.

22. Costa $V$, Angelini C, de Feis I, Ciccodicola A. Uncovering the complexity of transcriptomes with RNA-seq. Biomed Res Int. 2010. https://doi.org/10.1155/ 2010/853916.

23. Lu T, Lu G, Fan D, Zhu C, Li W, Zhao Q, Feng Q, Zhao Y, Guo Y, Li W, Huang $X$, Han B. Function annotation of the rice transcriptome at single-nucleotide resolution by RNA-seq. Genome Res. 2010;20(9):1238-49.

24. Wang Z, Fang B, Chen J, Zhang X, Luo Z, Huang L, Chen X, Li Y. De novo assembly and characterization of root transcriptome using Illumina pairedend sequencing and development of cSSR markers in sweetpotato (Ipomoea batatas). BMC Genomics. 2010;11:726.

25. Zhang K, Xia X, Zhang Y, Gan S. An ABA-regulated and Golgi-localized protein phosphatase controls water loss during leaf senescence in Arabidopsis. Plant J. 2012;69:667-78.

26. Kang YJ, Lee J, Kim YH, Lee S. Identification of tissue-specific gene clusters and orthologues of nodulation-related genes in Vigna angularis. Plant Genet Resour. 2014;12(S1):S21-6.

27. O'Rourke JA, Bolon Y, Bucciarelli B, Vance CP. Legume genomics: understanding biology through DNA and RNA sequencing. Annuls of Bot. 2014;113(7):1107-20.

28. Gailing O, Staton ME, Lane T, Schlarbaum SE, Nipper R, Owusu SA, Carlson JE. Construction of a framework genetic linkage map in Gleditsia triacanthos L. Plant Mol Biol Rep. 2017;35(2):177-87.

29. Lim PO, Kim HJ, Nam HG. Leaf senescence. Annu Rev Plant Biol. 2007;58: 115-36.

30. Guo Y, Gan S. Leaf senescence: signals, execution, and regulation. In: Schatten GP, editor. Current topics in developmental biology. New York: Academic Press; 2005. p. 83-112.

31. Yuan R. Effects of temperature on fruit thinning with ethephon in 'Golden delicious' apples. Sci Hortic-Amsterdam. 2007;113:8-12.

32. Zhu H, Dardick CD, Beers EP, Callanhan AM, Xia R, Yuan R. Transcriptomics of shading-induced and NAA-induced abscission in apple (Malus domestica) reveals a shared pathway involving reduced photosynthesis, alterations in carbohydrate transport and signaling and hormone crosstalk. BMC Plant Biol. 2011;11:138.

33. De Smet I, Signora L, Beeckman T, Inzé D, Foyer CH, Zhang H. An abscisic acid-sensitive checkpoint in lateral root development of Arabidopsis. Plant J. 2003;33:543-55.

34. Lefebvre V, North H, Frey A, Sotta B, Seo M, Okamoto M, Nambara E, Marion-Poll A. Functional analysis of Arabidopsis NCED6 and NCED9 genes indicates that ABA synthesized in the endosperm is involved in the induction of seed dormancy. Plant J. 2006;45:309-19.

35. Tan BC, Schwartz SH, Zeevaart JAD, McCarty DR. Genetic control of abscisic acid biosynthesis in maize. PNAS. 1997;94:12235-40.

36. Qin X, Zeevaart JAD. The 9-cis-epoxycarotenoid cleavage reaction is the key regulatory step of abscisic acid biosynthesis in water-stressed bean. PNAS. 1999;96:15354-61.

37. Tan B, Joseph LM, Deng W, Liu L, Li Q, Cline K, McCarty DR. Molecular characterization of the Arabidopsis 9-cis epoxycarotenoid dioxygenase gene family. Plant J. 2003;35:44-56.

38. Thompson AJ, Jackson AC, Symonds RC, Mulholland BJ, Dadswell AR, Blake PS, Burbidge A, Taylor IB. Ectopic expression of a tomato 9-cis-epoxycarotenoid dioxygenase gene causes over-production of abscisic acid. Plant J. 2000; 23(3):363-74

39. Schwartz SH, Qin X, Zeevaart JAD. Characterization of a novel carotenoid cleavage dioxygenase from plants. J Biol Chem. 2001; 276(27):25208-11.

40. Iuchi S, Kobayashi M, Taji T, Naramoto M, Seki M, Kato T, Tabata S, Kakubari Y, Yamaguchi-Shinozaki K, Shinozaki K. Regulation of drought tolerance by gene manipulation of 9-cis-epoxycarotenoid dioxygenase, a key enzyme in abscisic acid biosynthesis in Arabidopsis. Plant J. 2001;24(4):325-33.

41. Chang C, Stadler R. Ethylene hormone receptor action in Arabidopsis. BioEssays. 2001;23:619-27.

42. Chang C, Kwok SF, Bleecker AB, Meyerowitz EM. Arabidopsis ethyleneresponse gene ETR1: similarity of product to two-component regulators. Science. 1993;262(22):539-44.
43. Sakai H, Hua J, Chen QG, Chang C, Medrano LJ, Bleecker AB, Meyerowitz EM. ETR2 is an ETR1-like gene involved in ethylene signaling in Arabidopsis. PNAS. 1998;95:5812-7.

44. O'Malley RC, Rodriguez Fl, Esch JJ, Binder BM, O'Donnell P, Klee HJ, Bleecker $A B$. Ethylene-binding activity, gene expression levels, and receptor system output for ethylene receptor family members from Arabidopsis and tomato. Plant J. 2005:41:651-9.

45. Ulmasov T, Hagen G, Guilfoyle TJ. Activation and repression of transcription by auxin-response factors. PNAS. 1999;96:5844-9.

46. Ellis CM, Nagpal P, Young JC, Hagen G, Guilfoyle T, Reed JW. AUXIN RESPONSW FACTOR1 and AUXIN RESPONSE FACTOR2 regulate senescence and floral organ abscission in Arabidopsis thaliana. Development. 2005;132: 4563-74.

47. Nagpal P, Ellis CM, Weber H, Ploense SE, Barkawi LS, Guilfoyle TJ, Hagen G, Alonso JM, Cohen JD, Farmer EE, Ecker JR, Reed JW. Auxin response factors ARF6 and ARF8 promote jasmonic acid production and flower maturation. Development. 2005;132:4107-18

48. Fedoroff NV. Cross-talk in abscisic acid signaling. Sci Signal. 2002;140:10.

49. Mizuno T, Yamashino T. Comparative transcriptome of diurnally oscillating genes and hormone-responsive genes in Arabidopsis thaliana: insight into circadian clock-controlled daily responses to common ambient stresses in plants. Plant Cell Physiol. 2008:49(3):481-7.

50. Robertson FC, Skeffington AW, Gardner MJ, Webb AA. Interactions between circadian and hormonal signalling in plants. Plant Mol Biol. 2009;69:419-27.

51. Edwards KD, Akman OE, Knox K, Lumsden PJ, Thomson AW, Brown PE, Pokhilko A, Kozma-Bognar L, Nagy F, Rand DA, Millar AJ. Quantitative analysis of regulatory flexibility under changing environmental conditions. Mol Syst Biol. 2010;6:424

52. Umezawa T. Systems biology approaches to abscisic acid signaling. J Plant Res. 2011;124:539-48.

53. Grabherr MG, Haas BJ, Yassour M, Levin JZ, Thompson DA, Amit I, Adiconis X, Fan L, Raychowdhury R, Zeng Q, Chen Z, Mauceli E, Hacohen N, Gnirke A, Rhind N, di Palma F, Birren BW, Nusbaum C, Lindblad-Toh K, Friedman N, Regev A. Full-length transcriptome assembly from RNA-Seq data without a reference genome. Nat Biotechnol. 2011:29(7):644-52.

54. Haas BJ, Papanicolaou A, Yassour M, Grabherr M, Blood PD, Bowden J, Couger MB, Eccles D, Li B, Lieber M, MacManes MD, Ott M, Orvis J, Pochet N, Strozzi F, Weeks N, Westerman R, Wiliam T, Dewey CN, Henschel R, LeDuc RD, Friedman N, Friedman N, Regev A. De novo transcript sequence reconstruction from RNA-seq: reference generation and analysis with trinity. Nat Protoc. 2013;8(8):1494-521.

55. Guerriero G, Legay S, Hausman J. Alfalfa cellulose synthase gene expression under abiotic stress: a Hitchhiker's guide to RT-qPCR normalization. PLoS One. 2014;9(8):e103808.

56. Livak KJ, Schmittgen TD. Analysis of relative gene expression data using real-time quantitative PCR and the $2^{-\Delta \Delta C T}$ method. Methods. 2001:25:402-8.

Ready to submit your research? Choose BMC and benefit from:

- fast, convenient online submission

- thorough peer review by experienced researchers in your field

- rapid publication on acceptance

- support for research data, including large and complex data types

- gold Open Access which fosters wider collaboration and increased citations

- maximum visibility for your research: over $100 \mathrm{M}$ website views per year

At $\mathrm{BMC}$, research is always in progress.

Learn more biomedcentral.com/submissions 